\title{
INTRAOPERATIVE OBSERVATION OF CHANGES IN COCHLEAR NERVE ACTION POTENTIALS DURING EXPOSURE TO ELECTROMAGNETIC FIELDS GENERATED BY MOBILE PHONES
}

Corresponding Author:

Vittorio Colletti, MD, Chairman ENT Department, University of Verona, Verona, Italy Piazzale L. A. Scuro, 10; 37134 Verona. Tel: +390458124275; Fax: +39045802749.

e-mail: vittoriocolletti@yahoo.com

Marco Mandalà, MD, ENT Department, University of Verona, Verona, Italy

Paolo Manganotti, MD; Department of Neurology, University of Verona, Verona, Italy

Stefano Ramat, Phd, Department of Computer and Systems Science, University of Pavia, Pavia, Italy

Luca Sacchetto, MD, ENT Department, University of Verona, Verona, Italy

Liliana Colletti, Phd, ENT Department, University of Verona, Verona, Italy

Key words: electromagnetic field exposure, directly recorded cochlear nerve compound action potentials.

Word Count: 3743 


\begin{abstract}
The rapid spread of devices generating electromagnetic fields has raised concerns as to the possible effects of this technology on humans. The auditory system is the neural organ most frequently and directly exposed to electromagnetic activity due to the daily use of mobile phones. In recent publications, a possible correlation between mobile phone usage and central nervous system tumors has been detected. Very recently a deterioration of otoacoustic emissions and of the auditory middle latency responses after intensive and long term magnetic field exposure in humans has been demonstrated. To determine with objective observations if exposure to cell phone electromagnetic fields affects acoustically evoked cochlear nerve compound action potentials (CNAPs), 7 patients suffering from Ménière's disease and undergoing retrosigmoid vestibular neurectomy were exposed to the effects of mobile phone placed over the craniotomy for 5 minutes. All patients showed a substantial decrease in amplitude and a significant increase in latency of CNAPs during the 5 minutes of exposure to electromagnetic fields. These changes lasted for a period of around 5 minutes after exposure. The possibility that electromagnetic fields can produce relatively long-lasting effects on cochlear nerve conduction is discussed and analyzed in light of contrasting previous literature obtained under non-surgical conditions. Limitations of this novel approach, including the effects of the anesthetics, craniotomy and surgical procedure, are presented in detail.
\end{abstract}




\section{INTRODUCTION}

Mobile phones are today an integral part of modern life and allow people to maintain continuous communication without restraining the freedom of movement. The exponential diffusion of cell phones bears witness to the enormous success these devices have enjoyed in recent years. This enthusiasm is offset by the widespread opinion that cell phones may constitute a human health hazard. Public opinion therefore urgently seeks reassurance regarding the safety of the electromagnetic fields (EMF) produced by these devices. To date the scientific community has come up with discordant data on this issue.

In 1996 the World Health Organization set up the International Electromagnetic Fields Project to assess the existing scientific evidence of the possible health effects of electromagnetic fields, including radiofrequency fields emitted by mobile phones and their base stations, and to promote further research [1]. There are a number of experimental investigations showing that EMF exposure induces a series of alterations at the cellular level [2-5]. Changes in blood-brain-barrier permeability, neurotransmitter function and metabolic changes in brain tissues after exposure to low-intensity EMF have been found both in experimental animals [6-8] and humans [9-10]. Furthermore, it has recently been demonstrated that mobile phone bioactivity is related to its intensity or distance from the antenna: high for intensities down to less than $10 \mathrm{~mW} / \mathrm{cm}$ and still evident as far as $1 \mathrm{~mW} / \mathrm{cm}$ exhibiting 'window' effects [11].

Changes in neuronal electrophysiology, evoked potentials and EEG traces have been reported [12]. Varrò et al. [12] described a decrease in basic synaptic activity as a reduction in amplitude of evoked potentials in rat brain slice preparation when exposed to an electromagnetic field.

In humans, auditory brainstem responses (ABR) do not seem to be affected by mobile short term phone exposure, suggesting that mobile phone EMF do not affect the transmission of sensory stimuli from the cochlea to the midbrain [13-17]. However, exposure to mobile phone EMF does affect neural function in humans (when evaluating EEG recordings) as a function of exposure duration and its effect vanishes after phone use ceases [18]. Likewise, effects on human motor excitability have been reported using transcranial magnetic stimulation after EMF exposure [19]. 
From an epidemiological standpoint, Hardell et al. [20-21] found that exposure to mobile phone EMF correlated with an increased incidence of astrocytoma [20-21], acoustic neuroma and glioma [22] and in a recent review it was concluded that the "Current standard for exposure to microwaves during mobile phone use is not safe for long-term exposure and needs to be revised" [23]. Very recently, long term and intensive mobile phones users demonstrated a significantly higher risk of having both cochlear (absent otoacustic emission or higher speech frequency thresholds) and auditory cortex alterations (lower middle latency response waves amplitude) as compared to controls [24].

These conflicting data prompted us to investigate the effect of EMF exposure on cochlear nerve bioelectric activity using a near-field electrophysiological procedure.

It is known that the bioelectric activity of the cochlear nerve may be safely and reliably recorded during cerebellopontine angle surgery from the exposed human cochlear nerve [25-27].

The acoustically evoked cochlear compound nerve action potentials (CNAPs), directly recorded from the exposed nerve, are characterized by a stable morphology, amplitude and latency. The procedure is highly sensitive to damage to the cochlea and cochlear nerve and even minor stimulations, e.g. changes in temperature due to moderate cold or warm saline irrigation, cause significant transitory changes in latency of the potentials of the order of 0.2-0.3 ms that can be easily assessed [26-27].

The possibility of obtaining real-time recordings directly from the auditory nerve, the neural structure which is most likely to be directly affected by EMF generated by cell phones, prompted us to conduct the present study to investigate the effect of electromagnetic fields due to cell phone exposure on bioelectric activity in response to acoustic stimulation.

\section{MATERIALS AND METHODS}

The cohort reported here is a patient-based clinical population seen from January 2009 to March 2010. The study was conducted at the Department of Otolaryngology (tertiary referral center) of the University of Verona (Verona, Italy). All participants were affected by definite Ménière's disease (MD), according to the criteria of the American Academy of Otolaryngology-Head and Neck Surgery [1995]; they had also received 
medical therapy (diuretics, betahistine, low salt diet) for at least 6 months. All participants performed a complete audiological evaluation with pure tone audiometry, speech audiometry, impedance audiometry, ABR, electrocochleography (ECoG) and underwent a complete neuro-otological evaluation including: eye-movement bedside examination, vestibular evoked myogenic potentials (VEMPs), caloric test before the procedure and at follow-up examinations up to 6 months. A $1.5 \mathrm{~T}$ MRI evaluation of the brain was obtained before the procedure in all patients.

Twenty-one patients met the entry criteria. Six patients were not enrolled in the study due to profound hearing loss in the ear to be operated on. Three patients refused surgery. Seven patients underwent retrosigmoid vestibular neurectomy (VN) for disabling definite unilateral MD (classes $\mathrm{A}$ and $\mathrm{B}$ ) while being monitored for mobile phone EMF effects and 5 patients undergoing the same surgical procedure were enrolled in the study as a control group.

The 7 patients enrolled for EMF monitoring were first exposed for 5 minutes to the magnetic field generated by a cell phone during an active call placed over the craniotomy and then submitted to $\mathrm{VN}$ for Ménière's disease. Hearing was monitored intraoperatively using direct recording of CNAPs. The control group underwent 5 minutes' exposure in the same experimental conditions using the same mobile phone in stand-by mode.

All patients were duly informed regarding the aim and protocol of the experiment and gave their consent. The patients were operated on in the lateral position with the head rotated contralaterally to the operated ear. A retrosigmoid craniotomy with a diameter of 3-4 cm was carried out. The dura was opened in an " $\mathrm{H}$ " fashion. The cerebellum was gently depressed using a self-restraining retractor secured to the operating table. The entire area from the brainstem to the posterior wall of the petrous bone and the tentorium was exposed. Sharp dissection of the arachnoid allowed access to the eighth nerve.

Recording from the cochlear nerve was performed using a Teflon insulated silver electrode wire (Type Ag 7/10; Medwire Corporation, Mt. Vernon, NY, U.S.A.) with a small cotton wick sutured on its tip, which was uninsulated over a distance of 2-3 mm. The electrode was located on the proximal portion of the eighth nerve (Figure 1). 
A pledget of fibrin sponge covered the electrode in the CPA to stabilize and segregate it from the surgical field. The monopolar electrode (inverting electrode) was referenced to a subdermal platinum electrode (Type E-2; Grass Instrument Company, Quincy, MA, U.S.A.) placed in the ipsilateral tragus (non-inverting electrode). A subdermal ground electrode was positioned on the sternum.

To monitor auditory function (cochlea, cochlear nerve and cochlear nuclei) the ear undergoing the operation was stimulated by alternating click stimuli at 31 pulses/sec using a walkman-type earphone. Click intensity ranged from 100 to $120 \mathrm{~dB}$ sound pressure level, depending on the hearing level.

The recorded potentials were filtered through a 30 to $2,500-\mathrm{Hz}$ bandpass filter, amplified (10,000x or 20,000x) and averaged with a 10 channel evoked potential system (Medelec Synergy N-EP, ViasysTM). The acquired large-amplitude potentials were easily visualized after averaging a few responses (100 repetitions), providing information on the function of the cochlea and cochlear nerve with a delay of only 2-3 seconds.

CNAPs latency of the first negative peak (N1) and normalized absolute amplitude of N1 were evaluated in all subjects.

Prior to vestibular nerve section, a Nokia 6310i mobile phone was positioned over the craniotomy area. It was activated (ongoing call but no sound was played from the speaker of the mobile phone) or kept in stand-by mode respectively for the subjects and controls for 5 minutes. The distance between the mobile phone antenna and the eighth nerve was measured in every trial.

The mobile phone used in the present study emits and receives radio signals in the region of $900 \mathrm{MHz}$, and the highest specific absorption rate (SAR) value for this model when tested for compliance against the standard was $0.82 \mathrm{~W} / \mathrm{kg}$ (14).

To ensure the stability of the recording procedure and assess baseline behavior in all subjects, CNAPs were first recorded with the phone in stand-by mode over a 2 minute interval (T0), CNAPs were then continuously monitored throughout the entire 5 minutes of cochlear nerve exposure to the EMF (T1).

After the exposure to the cell phone, the potentials were recorded for 10 more minutes (T2) (time necessary to harvest an autologous abdominal fat graft) and surgery was then continued with the vestibular neurectomy. 
$\mathrm{ABR}$ were also recorded in all subjects at the same time as the CNAPs recordings. ABR were obtained using differential recordings from the vertex (non-inverting electrode) and the ipsilateral earlobe (inverting electrode) referenced to the sternum (ground). Needle electrodes were used to this end and the recorded activity was filtered (1000 to $2500 \mathrm{~Hz})$, amplified (100,000x) and averaged (100 sweeps).

Three months after surgery an audiometric evaluation with pure tone audiometry was performed to evaluate the extent of hearing preservation.

Recordings at time $\mathrm{T} 0$ and at the end of the 5 minutes exposure were compared with the CNAPs recordings obtained from the control group (sham exposure) at the same times.

Differences between the EMF and sham groups were tested using Student's t-test and a non-parametric test (Wilcoxon rank-sum test), as appropriate based on the results of the Kolmogorov-Smirnov test for normal distribution. A nonparametric repeated-measures ANOVA procedure (Friedman test) in combination with a post hoc test (Dunn's multiple-comparison test) was used to assess the effects of 5 minutes EMF or sham exposure by comparing the data collected at each minute of the 15 minutes of CNAPs recording with that recorded at T0. Statistical significance threshold was set at $\mathrm{p}=0.05$.

\section{RESULTS}

Demographic data of the population exposed to EMF and of the control group are reported in Table 1. No statistically significant differences in the distance measured from the mobile phone antenna to the eighth nerve were found between the study group and the control group ( $62 \pm 9 \mathrm{~mm}$ vs. $65 \pm 11 \mathrm{~mm} ; \mathrm{p}=0.6142$; t-test).

No significant changes could be detected with V wave latency of the ABR recordings with EMF exposure or sham exposure ( $p>0.05$; Wilcoxon test). Changes in wave I and III of the ABR could not be statistically analyzed due to their inconsistency in the recordings.

All CNAPs measurements showed stability and reproducibility at T0. A typical threephase response was obtained with a predominant negative peak (N1) generated by the depolarization wave propagation (Figure 2 and 3). The basal CNAPs latency and amplitude measures for both the study and control groups showed no significant changes at $\mathrm{T} 0$ ( $\mathrm{p}>0.05$, Wilcoxon test). 
During EMF exposure a significant decrease in CNAPs amplitude (Figure 3 and 4) and an increase in latency (Figure 3), which started in $\mathrm{T} 1$ and lasted, albeit to a minor extent, over the T2 period, were observed in all patients. Figure 2 shows representative series of CNAPs recorded in two subjects submitted to EMF exposure and sham exposure, respectively. The effect of the 5' EMF exposure over the 15' of recording, was shown to be statistically significant both on CNAPs latency and amplitude (Friedman test, $\mathrm{p}<0.0001$; Figure 4) compared to the baseline values recorded at T0. A post hoc test (Dunn's multiple-comparison test of the data recorded at each minute with that at T0) indicated that significant changes both in CNAPs latency and amplitude (Figure 4) could be demonstrated from few minutes after the beginning EMF exposure to around the $10^{\text {th }}$ minute of recording (5' after EMF exposure ceased).

A substantial deterioration in amplitude and latency of N1 was observed after 30-60 seconds of EMF exposure in all subjects. Due to the progressive deterioration of amplitude and latency of the potentials during exposure, we decided to limit the exposure to 5 minutes. This decision was taken because it is known that a shift in latency above $0.5 \mathrm{msec}$ may be critical for hearing preservation procedures during posterior fossa surgery [26,27]. Latency shifts above these values increase the chance of postoperative hearing deterioration proportionally to the extent of the changes in latency.

In our experiment the maximum latency shift was thus observed at 5 minutes of exposure with a mean increase in latency of $0.55 \pm 0.18 \mathrm{msec}$ (Table 1, Figure 4).

The same trend was observed in terms of amplitude reduction, the maximal amplitude change being observed at 5 minutes of exposure with a mean decrease in amplitude of $34 \pm 16 \%$ (Table 1, Figure 4). When comparing CNAPs at the end of EMF or sham exposure (T1), results showed statistically significant differences in terms of latency ( $p<.0001$; Wilcoxon test) and amplitude ( $p<.0273$; Wilcoxon test) between the EMFexposed subjects and the control group (Table 1).

Sham exposure to cell phones in stand-by mode on the 5 control subjects showed no significant differences in CNAPs latency and amplitude ( $p>0.05$; Friedman test).

The three-month audiological follow-up showed no changes ( $p>0.05$, Wilcoxon test) in hearing threshold when comparing the mean PTA of the whole population before $(35 \pm 12 \mathrm{~dB}$ HL) and after surgery $(42 \pm 9 \mathrm{~dB}$ HL). Furthermore, no statistically 
significant differences were found between the study group and the control group in terms of hearing changes $(p>0.05$, Wilconxon test) at the 3 -month examination.

\section{DISCUSSION}

The new finding of this study is that short-term exposure (5 minutes) to the EMF produced by active cell phones causes significant CNAPs deterioration, occurring with a specific time course, both during and after exposure. Changes are observed in CNAPs latency and amplitude, which respectively increased and decreased substantially and continuously during the 5-minute exposure. It was decided to suspend the exposure after 5 minutes to avoid possible permanent damage to the auditory structures. Altered latency and amplitude values persisted, albeit in milder forms, after removal of cell phone exposure. The largest changes were observed after 5 minutes of exposure and consisted in a $0.55 \pm 0.18 \mathrm{msec}$ latency increase and an $34 \pm 16 \%$ amplitude reduction. These changes were statistically significant $(\mathrm{p}<0.001)$. ABR analysis performed on the $\mathrm{V}$ wave showed no statistically significant differences related to the EMF exposure. Changes in wave I and III of the ABR could not be statistically analyzed due to their inconsistency in the recordings and this may be due to the experimental setting which required a limited number of stimuli (100) not to prolong the surgical time and to the amount of sensorineural hearing loss that all MD patients suffered from. Postoperative audiological follow-up revealed no significant hearing threshold deterioration in any of our patients.

The findings resulting from this novel approach to CNAPs seem in contrast with our ABR findings and with most of the literature results, showing no effect related to mobile phone exposure on pure tone audiometry, ABR, transient and distortion products otoacoustic emissions. However the population of the present study differs among the population study of the quoted papers in several aspects. We have analyzed subjects with a significant degree of hearing loss and within a surgical setting whereas in all other studies normal hearing subjects and non-surgical setting conditions [14-17,28,29]. Furthermore the ABR procedure of our study limited the number of stimuli to 100 to limit the surgical time. Nevertheless recent studies highlight trends for permanent abnormalities in several parameter of hearing function within long-term mobile phone 
users [30] and even a significantly higher risk for absent otoacustic emission and higher speech frequency thresholds [24].

The discrepancy between ABR and CNAPs results may be explained by different recording methodology and neurophysiological events. The direct recordings from the exposed cochlear nerve of CNAPs provide a real time measurement of extremely reliable, stable and relatively large amplitude signals. Therefore CNAPs are more sensitive to changes in the cochlea and/or cochlear nerve than ABR and offer functional information that heralds subclinical structural changes [26,27,31,32]. Despite ABR offers the opportunity to investigate peripheral and central auditory pathways, some features as the average technique, the low number of trials, the possible synchronization of volley in the central nuclei and the supramaximal stimulation performed in intraoperative monitoring may make the methodology less feasible and useful in testing the effect of EMF exposure on auditory pathways [33-34].

A series of additional factors may be responsible for the significant CNAPs deterioration with EMF exposure including anesthesia, increased cerebrospinal fluid shunting, volume intensity of the mobile phone, heat, metabolic changes and neurotransmitter alterations. The use of common anesthetics in surgery can produce a very low effect on the responses from auditory nerve fibers while it may impact the responses of the ascending auditory pathways form the cochlear nuclei [33]. CNAPs modifications may be also have been caused by an increased shunting of cerebrospinal fluid which could have accumulated around the recording electrode. However this possibility seems unlikely since the amplitudes were normalized after the end of EMF exposure, therefore increased shunting would not have affected the latency of the recorded CNAPs. Moreover, the volume intensity of the mobile phone could not have determined CNAPs changes since no sounds were played trough it and the external auditory canal was closed by the earphone and further covered by three sterile surgical drapes. Heat, on the other hand, might have been a contributing factor since the electrode placed on the auditory nerve could act as an antenna inducing local heating or local chemical reactions that could affect neural transmission. Yet, both these explanations appear unlikely since the temperature of cotton wick electrode measured before, during and after 5' of EMF exposure showed no changes and no Ag wires were in contact with the $8^{\text {th }}$ nerve. Recent studies based on a model of the human head during 
exposure to mobile phone EMF $[35,36]$ have shown a very low increase in temperature in the ear and brain regions close to the phone. Furthermore, it has been shown that the presence of a cochlear implant array inside the cochlea produces negligible variations in the averaged SAR values and consequently in temperature, both in the head and in the cochlear tissues [37].

The possibility that changes observed during EMF electromagnetic radiation could be due to interference with the stimulating and/or recording system was also considered. However, we found that the amplitude and latency shifts increased as a function of the duration of the exposure and slowly decreased after turning off the cell phone. Interference with the stimulating or recording system would have caused instantaneous and substantial modifications throughout the exposure period and should have ceased immediately after exposure. Similarly, possible acoustic interference, i.e. a masking effect, can also be ruled out.

Metabolic alteration in the cochlear nerve or at the synaptic level may be responsible for the CNAPs changes. RF fields generated by cell phones can affect membrane proteins and can change the movement of ions, especially the efflux of calcium ions from brain tissue across membranes under normal conditions [38]. This might cause subtle changes in cell function, but the significance of such effects for human health is uncertain. Thus, changes in calcium ion concentration might lead to alterations in neural functions. Other effects of mobile phone EMF on the nervous system are changes in blood-brain-barrier permeability due to hyperthermia, neurotransmitter functions, cellular metabolism and related electrophysiological modifications [39].

The available data suggest a complex reaction of the nervous system to EMF exposure. Other parameters to be considered in EMF exposure are frequency, duration, waveform and amplitude modulation, which are important determinants of biological responses and affect the dose-response relationship [11].

Despite evidence that mobile phone use can trigger brain evoked potentials [40] and induce CNAPs modifications, the crucial scientific question as to the pathophysiology of mobile-phone EMF effects on brain activity remains unanswered [41].

Two main limitations of the present investigation need to be discussed in detail. Due to the intraoperative recording methodology it is not, presently, possible to determine whether the EMF affect the cochlea, the cochlear nerve or both. CNAPs recordings 
(figure 2 and 3) showed a decrease in amplitude and a latency shift of all components, which suggest a decreased excitation of the auditory nerve. This finding suggests an effect of our stimulation on the cochlea rather than on the auditory nerve. A conduction block of the VII nerve would have determined an increase in the positive peak that precedes the N1 and a decrease in N1 amplitude. An effect on hair cells would explain the observed changes in the CNAPs [33].

The presence of a mild transitory threshold shift due to temporary cochlear damage would have no significant and long lasting effect on the latency and amplitude of the potentials recorded from the cochlear nerve [27]. Severe cochlear damage could certainly cause such effects. In this event, however, the time course of the effects would have been longer and a systematic postoperative hearing loss might have been observed. This did not occur in the studied population and postoperative shifts in the hearing threshold were not statistically different between the EMF exposure group and patients who underwent sham exposure.

Another limitation is the surgical access performed to investigate the eighth nerve. The retrosigmoid craniotomy directly exposes the eighth nerve to mobile phone EMF without any biological structure in-between. This condition is clearly far from reproducing the real-life EMF absorption of neural structures, because it offers a reliable measurement of the direct effect of a known EMF on a sensitive nerve without the interposition of other structures (skin, skull, fat, muscle, blood, grey and white matter) that have different SAR [17, 42]. All these structures play an important role in determining the SAR of inner ear structures in responses to EMF exposure [29].

The long-term effects of EMF exposure of the cochlear nerve could not be assessed in the present study. Nervous system conduction might adapt or severely deteriorate in response to the perturbation produced by repeated exposure to EMF.

Further investigations are needed to clarify these issues. Despite the fact that short-term exposure appears not to affect the human auditory system [43], audiologic abnormality trends have recently been described [24,30].

To the best of our knowledge, this study, for the first time, adds important information on the transitory and short-term effects of electromagnetic fields on the auditory nervous structures through intraoperative monitoring, and reveals relevant clinical and 
general health implications. Subjects undergoing VN for Ménière's disease represent a valuable and unique model for investigating human auditory physiopathology.

Further studies on larger numbers of patients with different experimental conditions are necessary to validate the present findings and clarify the pathological and physiological substrate of the described effect. 
Acknowledgments, Competing interests, Funding: nothing to declare

Copyright License Statement: The Corresponding Author has the right to grant on behalf of all authors and does grant on behalf of all authors, an exclusive license (or non-exclusive for government employees) on a worldwide basis to the BMJ Publishing Group Ltd and its Licensees to permit this article to be published in Journal of Neurology, Neurosurgery \& Psychiatry editions and any other BMJPGL products to exploit all subsidiary rights, as set out in our license http://jnnp.bmjjournals.com/ifora/licence.pdf. 
Table 1. Demographic data and latency and amplitude variations at the end of the 5' exposure for the EMF- exposed population and the control group.

\begin{tabular}{|c|c|c|c|c|c|}
\hline \multicolumn{1}{|c|}{ Group } & $\begin{array}{c}\text { Number } \\
\text { of } \\
\text { subjects }\end{array}$ & $\begin{array}{c}\text { Mean age } \\
\text { (years) }\end{array}$ & $\begin{array}{c}\text { Sex } \\
\text { (M/F) }\end{array}$ & $\begin{array}{c}\text { Latency shift } \\
\text { at the end of } \\
\text { 5' exposure } \\
\text { (ms) }\end{array}$ & $\begin{array}{c}\text { Normalized } \\
\text { amplitude } \\
\text { reduction at the } \\
\text { end of 5, } \\
\text { exposure (\%) }\end{array}$ \\
\hline $\begin{array}{l}\text { Study group } \\
\text { (EMF exposure) }\end{array}$ & 7 & $50.3 \pm 9.7$ & $4 / 3$ & $0.55 \pm 0.18$ & $34 \pm 16$ \\
\hline $\begin{array}{c}\text { Control group } \\
\text { (sham exposure) }\end{array}$ & 5 & $54.1 \pm 12.5$ & $2 / 3$ & $0.05 \pm 0.03$ & $98 \pm 2$ \\
\hline $\begin{array}{c}\text { Wilcoxon-Mann- } \\
\text { Whitney Test }\end{array}$ & & $\mathrm{p}=.5950$ & & $\mathrm{p}<0.0001$ & $\mathrm{p}<0.0001$ \\
\hline
\end{tabular}




\section{LEGENDS TO FIGURES}

Figure 1. Monopolar cotton-wick (arrow) electrode placed at the root entry zone of the eighth cranial nerve (asterisk) in a patient undergoing vestibular neurectomy via the retrosigmoid approach.

Figure 2. Series of CNAPs recorded in one of the patients submitted to EMF exposure and one control subject (sham exposure). CNAPs recordings obtained before, during and after EMF exposure are shown. A significant reduction in amplitude and increase in latency may be seen in the EMF exposure group.

T0, before EMF exposure; T1, during EMF exposure; T2, after EMF exposure.

Figure 3. Series of CNAPs recorded in one of the patients submitted to EMF exposure. Recordings obtained before RF exposure (dotted lines) are superimposed to CNAPs obtained during and after exposure (continuous lines). Significant reduction in amplitude and increase in latency can be seen.

T0, before EMF exposure; T1, during EMF exposure; T2, after EMF exposure.

Figure 4. Mean ( \pm SEM) values of latency shift and normalized amplitude variations from basal values in both groups of subjects. Patients submitted to EMF exposure show a significantly larger latency shift and amplitude reduction compared to controls. Results of the Friedman test $(*)$ in combination with a post hoc Dunn's multiplecomparison test are showed for the EMF exposure group. 


\section{REFERENCES}

1. WHO/OMS. Electromagnetic fields and public health. Mobile telephones and their base stations. Fact Sheet $n^{\circ} 193$ (1996).

2. Zhao R, Zhang S, Xu Z, et al. Studying gene expression profile of rat neuron exposed to $1800 \mathrm{MHz}$ radiofrequency electromagnetic fields with cDNA microassay. Toxicology. 2007;235:167-75.

3. Ammari M, Gamez C, Lecomte A, et al. GFAP expression in the rat brain following sub-chronic exposure to a $900 \mathrm{MHz}$ electromagnetic field signal. Int $\mathbf{J}$ Radiat Biol. 2010;86:367-75.

4. Campisi A, Gulino M, Acquaviva R, et al. Reactive oxygen species levels and DNA fragmentation on astrocytes in primary culture after acute exposure to low intensity microwave electromagnetic field. Neurosci Lett. 2010;473:52-5.

5. Brillaud E, Piotrowski A, de Seze R. Effect of an acute $900 \mathrm{MHz}$ GSM exposure on glia in the rat brain: a time-dependent study. Toxicology. 2007;238:23-33.

6. Nittby H, Brun A, Eberhardt J, et al. Increased blood-brain barrier permeability in mammalian brain 7 days after exposure to the radiation from a GSM-900 mobile phone. Pathophysiology. 2009;16:103-12.

7. Mausset-Bonnefont AL, Hirbec H, Bonnefont X, et al. Acute exposure to GSM 900-MHz electromagnetic fields induces glial reactivity and biochemical modifications in the rat brain. Neurobiol Dis. 2004;17:445-54.

8. Meral I, Mert $\mathrm{H}$, Mert $\mathrm{N}$, et al. Effects of $900-\mathrm{MHz}$ electromagnetic field emitted from cellular phone on brain oxidative stress and some vitamin levels of guinea pigs. Brain Res. 2007;1169:120-4.

9. Aalto S, Haarala C, Brück A, et al. Mobile phone affects cerebral blood flow in humans. J Cereb Blood Flow Metab. 2006;26:885-90.

10. Söderqvist F, Carlberg M, Hansson Mild K, et al. Exposure to an $890-\mathrm{MHz}$ mobile phone-like signal and serum levels of S100B and transthyretin in volunteers. Toxicol Lett. 2009;189:63-6.

11. Panagopoulos DJ, Chavdoula ED, Margaritis LH. Bioeffects of mobile telephony radiation in relation to its intensity or distance from the antenna. Int $\mathbf{J}$ Radiat Biol. 2010;86:345-57. 
12. Varró P, Szemerszky R, Bárdos G, et al. Changes in synaptic efficacy and seizure susceptibility in rat brain slices following extremely low-frequency electromagnetic field exposure. Bioelectromagnetics. 2009;30:631-40.

13. Arai N, Enomoto H, Okabe S, et al. Thirty minutes mobile phone use has no short-term adverse effects on central auditory pathways. Clin Neurophysiol. 2003;114:1390-4.

14. Oysu C, Topak M, Celik O, et al. Effects of the acute exposure to the electromagnetic field of mobile phones on human auditory brainstem responses. Eur Arch Otorhinolaryngol. 2005;262:839-43.

15. Stefanics G, Kellényi L, Molnár F, et al. Short GSM mobile phone exposure does not alter human auditory brainstem response. BMC Public Health. $2007 ; 7: 325$.

16. Balbani AP, Montovani JC. Mobile phones: influence on auditory and vestibular systems. Braz J Otorhinolaryngol. 2008;74:125-31.

17. Kwon MS, Jääskeläinen SK, Toivo $\mathrm{T}$, et al. No effects of mobile phone electromagnetic field on auditory brainstem response. Bioelectromagnetics. 2010;31:48-55.

18. Croft RJ, Chandler JS, Burgess AP, et al. Acute mobile phone operation affects neural function in humans. Clin Neurophysiol. 2002;113:1623-32.

19. Brignani D, Manganotti P, Rossini PM, et al. Modulation of cortical oscillatory activity during transcranial magnetic stimulation. Hum Brain Mapp. 2008;29:603-12.

20. Hardell L, Carlberg M, Söderqvist F, et al. Long-term use of cellular phones and brain tumours: increased risk associated with use for $>$ or $=10$ years. Occup Environ Med. 2007;64:626-32.

21. Hardell L, Carlberg M. Mobile phones, cordless phones and the risk for brain tumours. Int J Oncol. 2009;35:5-17.

22. Hardell L, Carlberg M, Söderqvist F, et al. Meta-analysis of long-term mobile phone use and the association with brain tumours. Int J Oncol. 2008;32:1097103. 
23. Hardell L, Carlberg M, Hansson Mild K. Epidemiological evidence for an association between use of wireless phones and tumor diseases. Pathophysiology. 2009;16:113-22.

24. Modi R, Virk R, Munjal S. Auditory Changes in Mobile Users: Is Evidence Forthcoming? Otol Head \& Neck Surg 2010;143:P85-P86.

25. Moller AR, Jannetta PJ. Compound action potentials recorded intracranially from the auditory nerve in man. J Exp Neurol 1981;74:862-874.

26. Colletti V, Fiorino FG. Vulnerability of hearing function during acoustic neuroma surgery, Acta Otolaryngol 1994;114:264-270.

27. Colletti V, Fiorino FG, Sacchetto L. Iatrogenic damage of hearing during surgery for acoustic neuroma Skull Base Surg 1996;6:153-161.

28. Mora R, Crippa B, Mora F, et al. A study of the effects of cellular telephone microwave radiation on the auditory system in healthy men. Ear Nose Throat J. 2006; 85:160-163.

29. Parazzini M, Brazzale AR, Paglialonga A, et al. Effects of GSM cellular phones on human hearing: the European project "GUARD". Radiat Res. 2007;168:608613.

30. Panda NK, Jain R, Bakshi J, et al. Audiologic disturbances in long-term mobile phone users. J Otolaryngol Head Neck Surg. 2010;39:5-11.

31. Moller AR, Colletti V, Fiorino FG. Click evoked responses from the exposed intracranial portion of the eighth nerve during vestibular nerve section: bipolar and monopolar recordings. Electroencephal Clin Neurophysiol (Amsterdam), 1994;92:17-29.

32. Yamakami I, Yoshinori H, Saeki N, et al. Hearing preservation and intraoperative auditory brainstem response and cochlear nerve compound action potential monitoring in the removal of small acoustic neurinoma via the retrosigmoid approach. J Neurol Neurosurg Psychiatry. 2009;80:218-27.

33. Møller, A.R. Hearing: Anatomy, Physiology, and Disorders of the Auditory System, 2nd Ed. Academic Press, Elsevier Amsterdam, 2006.

34. Popper AN, Fay RR. The Mammalian Auditory Pathway: Neurophysiology. Springer-Verlag, 1991. 
35. Chen HY, Yang HP. Temperature increase in human heads due to different models of cellular phones. Electromagnetics 2006;26:439-459.

36. Schmid G, Uberbacher R, Samaras T, et al. High-resolution numerical model of the middle and inner ear for a detailed analysis of radio frequency absorption. Phys Med Biol. 2007;52:1771-81.

37. Sibella F, Parazzini M, Paglialonga A, et al. Assessment of SAR in the tissues near a cochlear implant exposed to radiofrequency electromagnetic fields. Phys Med Biol. 2009;54:N135-41.

38. Maskey D, Kim M, Aryal B, et al. Effect of $835 \mathrm{MHz}$ radiofrequency radiation exposure on calcium binding proteins in the hippocampus of the mouse brain. Brain Res. 2010;1313:232-41.

39. Valentini E, Curcio G, Moroni F, et al. Neurophysiological effects of mobile phone electromagnetic fields on humans: a comprehensive review. Bioelectromagnetics. 2007;28:415-32.

40. Marino AA, Carrubba S. The effects of mobile-phone electromagnetic fields on brain electrical activity: a critical analysis of the literature. Electromagn Biol Med. 2009;28:250-74.

41. Carrubba S, Frilot C 2nd, Chesson AL Jr, et al. Mobile-phone pulse triggers evoked potentials. Neurosci Lett. 2010;469:164-8.

42. Gajsek P, Hurt WD, Ziriax JM, et al. Parametric dependence of SAR on permittivity values in a man model. IEEE Trans Biomed Eng. 2001;48:1169-77.

43. Parazzini M, Sibella F, Lutman ME, et al. Effects of UMTS cellular phones on human hearing: results of the European project EMFnEAR. Radiat Res. 2009;172:244-51. 


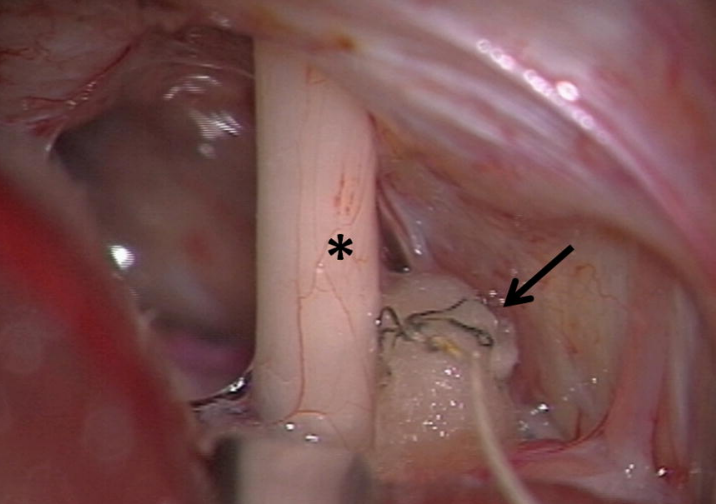




\section{EMF exposure}

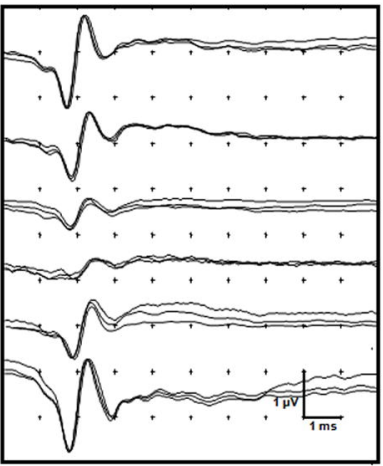

\section{SHAM exposure}

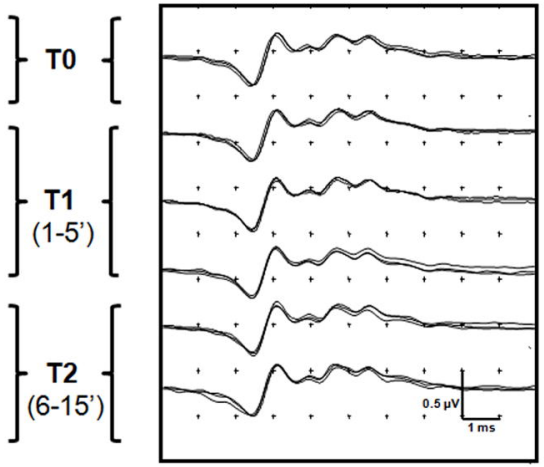




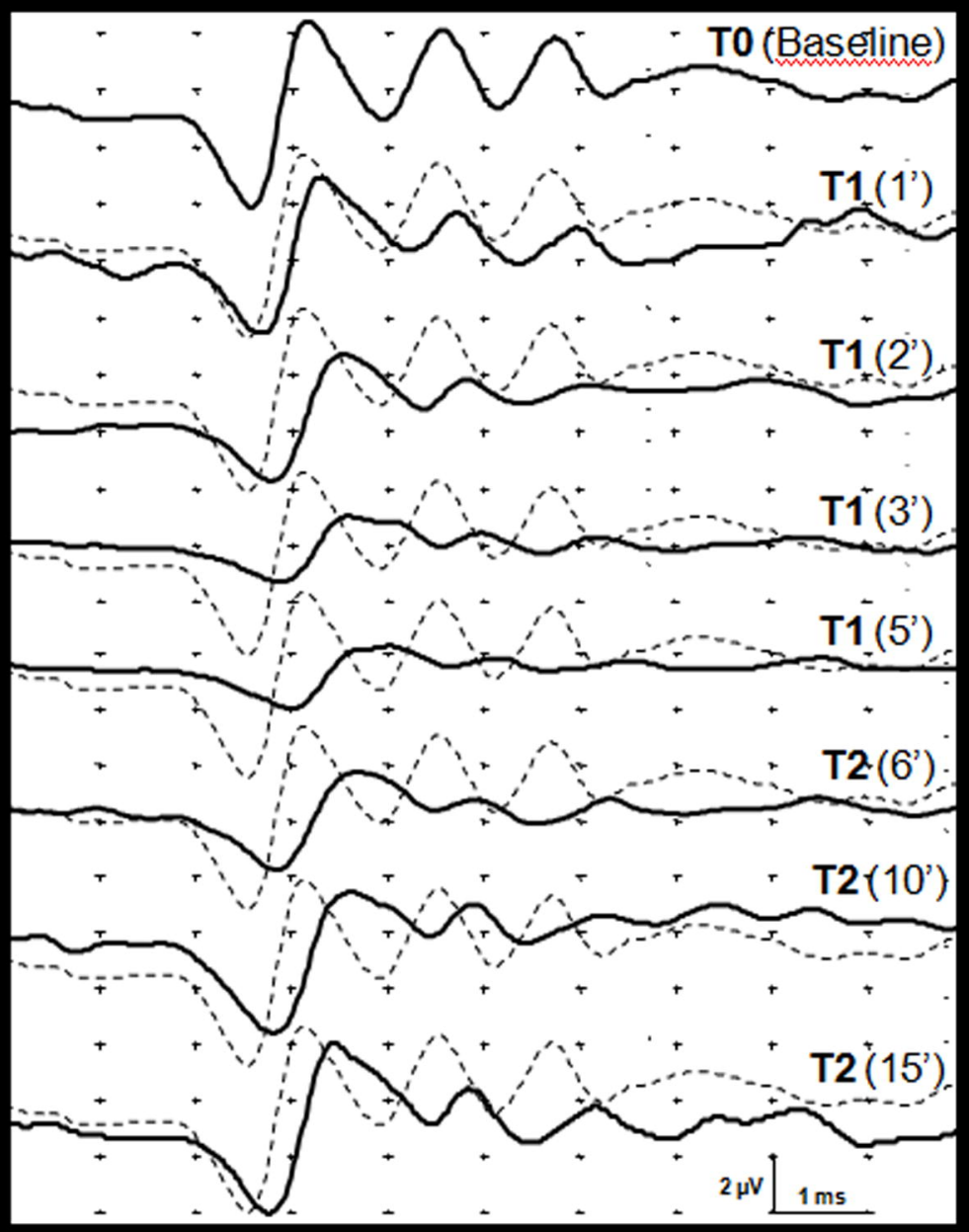




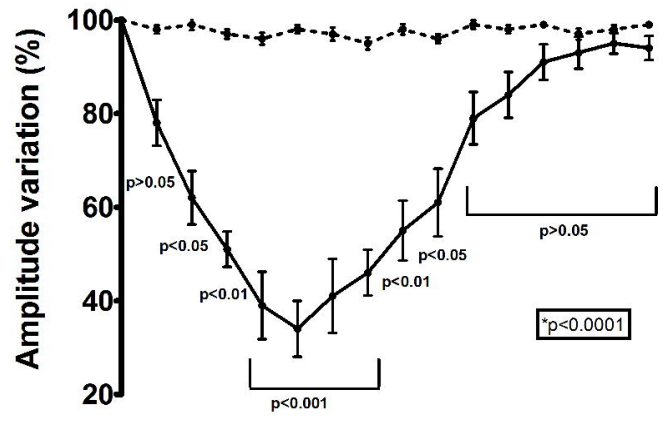

- EMF exposure
- Sham exposure

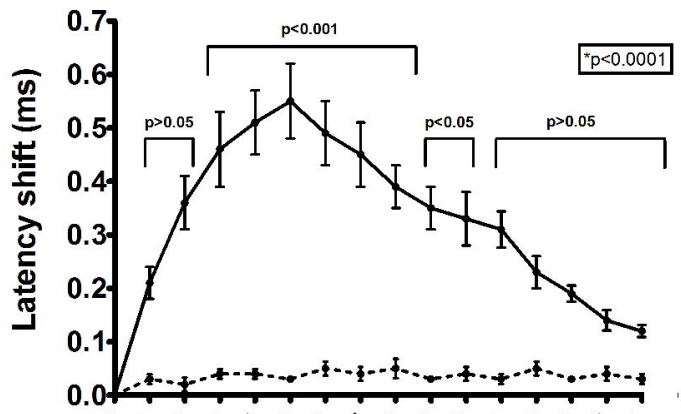

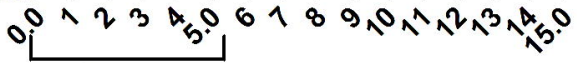
Exposure Minutes 